\title{
EDITORIAL
}

\section{Serotonergic neurons derived from induced pluripotent stem cells (iPSCs): a new pathway for research on the biology and pharmacology of major depression}

\section{Molecular Psychiatry (2016) 21, 1-2; doi:10.1038/mp.2015.201}

Chronic mental illness has a major and escalating personal, social and economic cost. The burden of mental, neurological and substance use (MNS) disorders increased by $41 \%$ between 1990 and 2010, and these disorders now account for 1 of every 10 lost years of health globally. ${ }^{1}$ It has been estimated that in the United States $5 \%$ of ambulatory care visits involve patients with a diagnosis of a mental health disorder; most of these are classified as depression, psychoses, or anxiety disorders. ${ }^{2}$ The annual cost of depression in the United States is estimated to be $\$ 210$ billion- $45 \%$ attributable to direct costs, $5 \%$ to suicide-related costs and $50 \%$ to workplace costs. ${ }^{3}$ In Australia, $24 \%$ of disease burden is caused by mental disorders, with a long-term impact on productivity and health outcomes, the largest component being due to depression. The cost of mental illness to Australia is $\$ 190$ billion a year, about $12 \%$ of the economy's annual output. For every dollar allocated to mental health services in the specialized sector, an additional $\$ 3.10$ is spent on providing support services to people who require assistance due to mental illness. There is a mortality gap-20 years for men, 15 for women-between people with and without mental illness in high-income countries, ${ }^{4}$ one of the greatest civil rights scandals of our time. It is imperative that new approaches be undertaken to elucidate the underlying biology and treat mental illness, including depression, much more effectively.

A major challenge in the study of mental illness is the fact that they are disorders of brain tissue, which lies within a bone helmet (the skull) and cannot be biopsied for studies. The bread and butter of medical research-tissue biopsy to examine diseased cells-has not been available for psychiatric investigation. We can only imagine how far behind cancer research would be if we could not biopsy cancer and grow it in culture. Induced pluripotent stem cells (iPSCs) represent a major scientific breakthrough that circumvents that obstacle. Briefly, cells in the body differentiate forward from stem cells, such as those in the egg and sperm, to specialized tissues, such as brain, heart, bone and muscle. In nature, such differentiation is a one-way street; tissues do not go backward from specialized to stem cells.

However, revolutionary methods have been developed that allow cells to dedifferentiate from specialized cells backward into stem cells, known as induced pluripotent stem cells (iPSCs). The iPSC technology was pioneered by Shinya Yamanaka's lab in Kyoto, Japan, which showed in 2006 that the introduction of four specific genes encoding transcription factors could convert adult cells into pluripotent stem cells. ${ }^{5}$ Specifically, they demonstrated induction of pluripotent stem cells from adult mouse fibroblasts by introducing four factors-Oct3/4, Sox2, c-Myc and Klf4-under embryonic stem (ES) cell culture conditions. These cells, which they designated induced pluripotent stem cells, exhibited the morphology and growth properties of ES cells and express ES cell marker genes. Following injection into blastocysts, iPSCs contributed to mouse embryonic development. These findings demonstrate that pluripotent stem cells can be directly generated from fibroblast cultures via the addition of only a few defined factors. Along with Sir John Gurdon, Shinya Yamanaka was awarded the 2012 Nobel Prize in Physiology or Medicine 'for the discovery that mature cells can be reprogrammed to become pluripotent.' Those iPSCs can be differentiated into various cell types in tissue culture.

In 2011, Fred (Rusty) Gage and colleagues at the Salk Institute in California showed that skin cells from patients with schizophrenia could be dedifferentiated in vitro into iPSCs and then differentiated into dopaminergic neurons, which are the neurons thought to be dysregulated in schizophrenia and that are also the target for drugs that treat schizophrenia. ${ }^{6}$ That landmark work, by Brennand et al., showed impairments in neurons derived from schizophrenic individuals, as compared with those from healthy controls. The neurons that they generated from iPSCs derived from schizophrenia patients (SCZD hiPSCs) showed diminished neuronal connectivity in conjunction with decreased neurite numbers, PSD95-protein levels and glutamate receptor expression. Gene expression profiles of SCZD hiPSC neurons identified altered expression of many components of the cyclic AMP and WNT signaling pathways. Moreover, Brennand and colleagues found that key cellular and molecular elements of the schizophrenia phenotype were ameliorated following treatment of SCZD hiPSC neurons with the antipsychotic loxapine. Such a novel approach makes this method ideal for testing individual responses to various medications, without having to expose patients to multiple drugs to determine which ones may work best for that person. Since 2011, a substantial body of work has been generated on iPSCs generated from patients with psychiatric disorders, including schizophrenia, ${ }^{7-10}$ bipolar disorder, ${ }^{11}$ and MECP2 duplication syndrome (see the cover article of the next issue of Molecular Psychiatry, 21.2, 2016), ${ }^{12}$ as well as in a Rett syndrome model in Mecp2-deficient mice, ${ }^{13}$ leading to an entirely new approach to molecular psychiatry.

As an example of the power of this method, in November 2015 Mertens et al reported in Nature their investigation of the cellular phenotypes of hippocampal dentate gyrus-like neurons derived from iPSCs of patients with bipolar disorder. Guided by RNA sequencing expression profiling, they detected mitochondrial abnormalities in young neurons from bipolar disorder patients; in addition, using both patch-clamp recording and somatic $\mathrm{Ca}^{2+}$ imaging, they observed hyperactive action-potential firing. This hyperexcitability phenotype of young neurons in bipolar disorder was selectively reversed by lithium treatment, but importantly only in neurons derived from patients who also responded to lithium treatment. Based on their exciting new data they propose that hyperexcitability is an early endophenotype of bipolar disorder, and suggest that their model may be useful in developing new treatments. What is exceptionally remarkable in that work is the fact that the neurons derived from iPSCs responded or not in culture to lithium treatment according to the ability of the patients from which the iPSCs originated to respond to that treatment. This further validates the iPSC approach as an early testing method to identify new drugs for psychiatric disorders. $^{14}$ 
Two separate research groups have now substantially advanced iPSC research, making it possible to bring it to the domain of depression by generating, through the iPSC approach, serotonergic neurons that can now be studied in vitro. Previously, serotonergic neurotransmission could be studied only in animal models.

Jian Feng and his team in Buffalo, New York, and Shanghai, China, and Fred Gage's team produced two independent papers, appearing in this issue of Molecular Psychiatry, ${ }^{15,16}$ that show how iPSC cells can be harnessed to generate serotonergic neurons. Feng's group, in the paper by Xu et al., which is highlighted on the cover of this issue of Molecular Psychiatry, found that human primary fibroblasts were directly converted to induced serotonergic (i5HT) neurons by the expression of Ascl1, Foxa2, Lmx1b and FEV. The transdifferentiation was enhanced by p53 knockdown and appropriate culture conditions, including hypoxia. They elegantly determined that $\mathrm{i} 5 \mathrm{HT}$ neurons expressed markers for mature serotonergic neurons, had $\mathrm{Ca}^{2+}$-dependent $5 \mathrm{HT}$ release and selective $5 \mathrm{HT}$ uptake, and exhibited spontaneous action potentials and spontaneous excitatory postsynaptic currents. Moreover, application of serotonin significantly increased the firing rate of spontaneous action potentials, demonstrating the functional utility of i5HT neurons for studying serotonergic neurotransmission. $^{15}$

In a separate line of work, Vadodaria et al. ${ }^{16}$ in Fred Gage's lab showed that overexpressing the transcription factors NKX2.2, FEV, GATA2 and LMX1B in combination with ASCL1 and NGN2 directly and efficiently generated serotonergic neurons from human fibroblasts. Induced serotonergic neurons, which they call iSNs (in contrast to $\mathrm{Xu}$ and colleagues' designation as 'i5HT'), showed increased expression of specific serotonergic genes known to be expressed in raphe nuclei. iSNs displayed spontaneous action potentials, released serotonin in vitro and functionally responded to selective serotonin reuptake inhibitors (SSRIs). This approach is highlighted in our Image section in this issue.

It is absolutely remarkable that we can simultaneously publish in this single issue of our journal two independent approaches to the generation of serotonergic neurons from iPSCs. Serotonin neurons are dysregulated in depression and are the target of commonly used antidepressants, particularly the SSRIs, such as fluoxetine, sertraline, citalopram, paroxetine, escitalopram, dapoxetine, mesembrine, seproxetine, and zimelidine. Depression has never before been studied in this manner. These new methods will now make it possible to study serotonergic neurons from depressed patients, as well as from those with other disorders thought to involve serotonin neurotransmission, such as anxiety disorders, obsessive-compulsive disorder, autism and eating disorders. The exciting papers by Xu et al. ${ }^{15}$ and Vadodaria et $a .^{16}$ will certainly open a new avenue for research relevant to major depression and other psychiatric disorders.

\section{CONFLICT OF INTEREST}

The authors declare no conflict of interest.

J Licinio and M-L Wong

Mind and Brain Theme, South Australian Health and Medical Research Institute, and Department of Psychiatry, School of Medicine, Flinders University, Adelaide, Australia E-mail: julio.licinio@sahmri.com

\section{REFERENCES}

1 Patel V et al. Lancet 2015; 3rd edn (e-pub ahead of print 7 October 2015).

2 Reeves WC et al. MMWR Surveill Summ 2011; 60 (Suppl 3): 1-29.

3 Greenberg PE et al. J Clin Psychiatry 2015; 76: 155-162.

4 Thornicroft G. Br J Psychiatry 2011; 199: 441-442.

5 Takahashi K et al. Cell 2006; 126: 663-676.

6 Brennand KJ et al. Nature 2011; 473: 221-225.

7 Hartley BJ et al. Mol Psychiatry 2015; 20: 549-550.

8 Brennand K et al. Mol Psychiatry 2015; 20: 361-368.

9 Topol A et al. Transl Psychiatry 2015; 5: e662.

10 Robicsek O et al. Mol Psychiatry 2013; 18: 1067-1076.

11 Madison JM et al. Mol Psychiatry 2015; 20: 703-717.

12 Nageshappa S et al. Mol Psychiatry 2015 (e-pub ahead of print 8 September 2015).

13 Farra N et al. Mol Psychiatry 2012; 17: 1261-1271.

14 Mertens J et al. Nature 2015; 527: 95-99.

15 Xu Z et al. Mol Psychiatry 2016; 21: 62-70.

16 Vadodaria KC et al. Mol Psychiatry 2016; 21: 49-61. 